\title{
Comment on: Nathan DM, Buse JB, Davidson MB et al. (2006) Management of hyperglycaemia in type 2 diabetes: a consensus algorithm for the initiation and adjustment of therapy. A consensus statement from the American Diabetes Association and the European Association for the Study of Diabetes. Diabetologia 49: 1711-1721
}

\author{
T. Takamura • A. Shimizu $•$ H. Ando $\cdot$ S. Kaneko
}

Received: 3 October 2006 / Accepted: 3 October 2006 / Published online: 23 November 2006

(C) Springer-Verlag 2006

Glucose-responsive insulin secretion is frequently impaired prior to the onset of type 2 diabetes [1]. In addition, considering the reduced pancreatic beta cell mass in patients with type 2 diabetes [2], defects in insulin secretion appear heterogeneous in this patient group. In some patients, only postprandial insulin secretion appears impaired, whilst in others, basal insulin secretion is also affected. However, postprandial insulin secretion is impaired in almost all patients with type 2 diabetes.

In the recently published consensus statement from the American Diabetes Association [3] and the European Association for the Study of Diabetes [4] concerning the initiation and adjustment of therapy for managing hyperglycaemia in type 2 diabetes, initial insulin therapy is aimed at increasing basal insulin supply with intermediate- or long-acting insulins. Such a regimen cannot completely mimic a physiological pattern of insulin secretion. Consequently, postprandial glycaemic control tends to be inadequate, and a risk exists of fasting hypoglycaemia. Furthermore, the postprandial hyperglycaemia observed in patients with early-stage or mild type 2 diabetes increased the risk of microangiopathy or arteriosclerosis in two largescale clinical studies of type 2 diabetes, the United Kingdom Prospective Diabetes Study [5] and the DECODE study [6]. It has also been suggested that therapy focused on lowering postprandial glucose, rather than fasting

T. Takamura $(\bowtie) \cdot$ A. Shimizu $\cdot$ H. Ando $\cdot$ S. Kaneko

Department of Disease Control and Homeostasis,

Kanazawa University Graduate School of Medical Science,

13-1 Takara-machi,

Kanazawa, Ishikawa 920-8641, Japan

e-mail: ttakamura@m-kanazawa.jp glucose, may be superior for lowering $\mathrm{HbA}_{1 \mathrm{c}}$ [7] and glucose variability (spike) [8]. Insulin preparations have been developed to provide insulin replacement that more closely reflects physiological insulin secretion; the introduction of rapid-acting insulin analogues and long-acting soluble insulin analogues has been a further step in this direction. Rapid-acting insulin analogues have been particularly effective at targeting postprandial hyperglycaemia [8]. They can, therefore, effectively reduce postprandial plasma glucose, even if administered immediately before a meal, and also minimise the risk of hypoglycaemia prior to the next meal [8].

The exact contribution of postprandial and fasting glucose increments to overall hyperglycaemia remains controversial. Monnier et al. reported that postprandial glucose excursion contributes up to $\sim 70 \%$ of the glucose load in patients with diabetes whose $\mathrm{HbA}_{1 \mathrm{c}}$ levels are $<7.3 \%$, whereas the contribution of fasting hyperglycaemia increases gradually as diabetes worsens [9]. However, even in patients whose glycaemic control is poor, studies have demonstrated that treatment targeting postprandial hyperglycaemia, which improves glucose-responsive insulin secretion in patients with glucose desensitisation, improves both postprandial and also morning fasting plasma glucose (FPG) in such patients [10-12].

We hypothesised that mealtime dosing with rapid-acting insulin analogues strictly controls postprandial and daytime plasma glucose levels, and thereby improves glucose desensitisation and glucose-responsive insulin secretion. To test these hypotheses, we recently evaluated the effect of a mealtime, rapid-acting insulin analogue on the minimum early morning FPG levels (nadir FPG) in 40 Japanese 
patients with type 2 diabetes whose existing antidiabetic medication was discontinued [12]. Approximately half $(52.5 \%)$ of the patients achieved a nadir FPG of $<6.7 \mathrm{mmol} / 1$ with mealtime dosing of a rapid-acting insulin analogue alone. No basal insulin replacement was needed in these patients. Patients, on the other hand, whose FPG levels remained high, even after postprandial hyperglycaemia improved, were presumed to have defects in basal insulin secretion [12]. To further confirm our finding, we prospectively followed 160 patients with type 2 diabetes treated with mealtime dosing of a rapid-acting insulin analogue. After 1 year of treatment at our out-patient clinic, about $60 \%$ of these patients were able to continue this therapy successfully; FPG was $6.4 \pm 1.6 \mathrm{mmol} / 1$ and $\mathrm{HbA}_{1 \mathrm{c}}$ was $6.5 \pm 1.1 \%$, compared with FPG of $6.0 \pm 1.2 \mathrm{mmol} / 1$ and $\mathrm{HbA}_{1 \mathrm{c}}$ of $6.9 \pm 1.1 \%$ in the patients who required additional basal insulin replacement (Takamura et al., unpublished observation). This suggests that basal insulin secretion was maintained in these patients, and only prandial insulin secretion was impaired. For such patients, glycaemic control achieved with sulfonylureas, which continuously promote insulin secretion, or with a long-acting insulin, may increase the risk of fasting hypoglycaemia. It may also contribute to obesity as a result of differentiation or hyperplasia of adipocytes during episodes of hyperinsulinaemia. Such patients may benefit instead from treatment with rapid-acting insulin analogue preparations, which more closely mimic physiological insulin secretion. These formulations may reduce the risk of hypoglycaemia by achieving a better balance between glucose and insulin levels, and also minimise increases in obesity, which might otherwise result from persistent hyperinsulinaemia during periods of fasting or after meals.

Based on these findings, we propose mealtime dosing with a rapid-acting insulin analogue as an initial insulin regimen for patients with type 2 diabetes. This regimen may be superior in lowering postprandial glucose excursion and the glucose spike, which are cardiovascular risk factors. The regimen may also help reduce the risk of hypoglycaemia and obesity associated with insulin therapy. Because not only postprandial, but also FPG can be modestly reduced by this regimen, the requirement for basal insulin replacement should be considered only in patients who do not achieve good FPG levels thereafter.
We suggest that further discussion is needed for better care of patients with type 2 diabetes.

\section{References}

1. Kadowaki T, Miyake Y, Hagura R et al (1984) Risk factors for worsening to diabetes in subjects with impaired glucose tolerance. Diabetologia 26:44-49

2. Kahn SE (2001) Clinical review 135: the importance of beta-cell failure in the development and progression of type 2 diabetes. J Clin Endocrinol Metab 86:4047-4058

3. Nathan DM, Buse JB, Davidson MB et al (2006) Management of hyperglycemia in type 2 diabetes: a consensus algorithm for the initiation and adjustment of therapy: a consensus statement from the American Diabetes Association and the European Association for the Study of Diabetes. Diabetes Care 29:1963-1972

4. Nathan DM, Buse JB, Davidson MB et al (2006) Management of hyperglycaemia in type 2 diabetes: a consensus algorithm for the initiation and adjustment of therapy. A consensus statement from the American Diabetes Association and the European Association for the Study of Diabetes. Diabetologia 49:1711-1721

5. Stratton IM, Adler AI, Neil HA et al (2000) Association of glycaemia with macrovascular and microvascular complications of type 2 diabetes (UKPDS 35): prospective observational study. BMJ 321:405-412

6. DECODE Study Group, the European Diabetes Epidemiology Group (2001) Glucose tolerance and cardiovascular mortality: comparison of fasting and 2-hour diagnostic criteria. Arch Intern Med 161:397-405

7. Bastyr EJ 3rd, Stuart CA, Brodows RG et al (2000) Therapy focused on lowering postprandial glucose, not fasting glucose, may be superior for lowering HbA1c. IOEZ Study Group. Diabetes Care 23:1236-1241

8. Del Prato S (2002) In search of normoglycaemia in diabetes: controlling postprandial glucose. Int J Obes Relat Metab Disord 26 (Suppl 3):S9-S17

9. Monnier L, Lapinski H, Colette C (2003) Contributions of fasting and postprandial plasma glucose increments to the overall diurnal hyperglycemia of type 2 diabetic patients: variations with increasing levels of $\mathrm{HbA}_{1 \mathrm{c}}$. Diabetes Care 26:881-885

10. Feinglos MN, Thacker CH, English J, Bethel MA, Lane JD (1997) Modification of postprandial hyperglycemia with insulin lispro improves glucose control in patients with type 2 diabetes. Diabetes Care 20:1539-1542

11. Saloranta C, Hershon K, Ball M, Dickinson S, Holmes D (2002) Efficacy and safety of nateglinide in type 2 diabetic patients with modest fasting hyperglycemia. J Clin Endocrinol Metab 87:41714176

12. Takamura T, Sakurai M, Nakamura M, Shimizu A, Ando H, Kaneko S (2006) Factors associated with improvement of fasting plasma glucose level by meal-time dosing of a rapid-acting insulin analog in type 2 diabetes. Diabetes Res Clin Pract. DOI 10.1016/ j.diabres.2006.07.019 